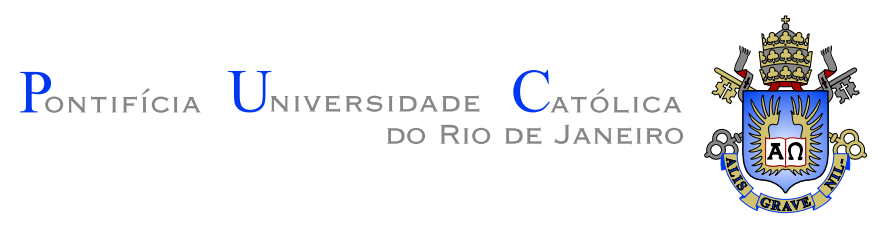

Franklin Antonio Sanchez Paiba

\title{
Códigos Fontanais Bidimensionais para Canais com Apagamento
}

Dissertação apresentada como requisito parcial para obtenção do grau de Mestre pelo Programa de Pós-graduação em Engenharia Elétrica do Departamento de Engenharia Elétrica da PUC-Rio

Orientador: Prof. Weiler Alves Finamore 


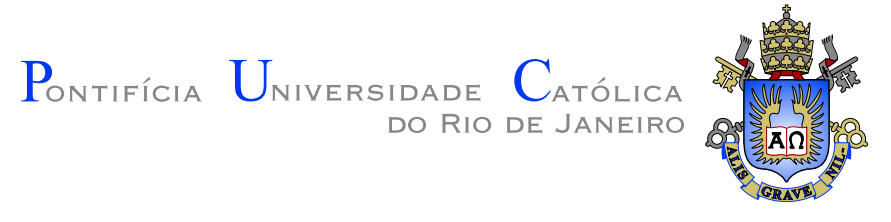

Franklin Antonio Sanchez Paiba

\title{
Códigos Fontanais Bidimensionais para Canais com Apagamento
}

\begin{abstract}
Dissertação apresentada como requisito parcial para obtenção do grau de Mestre pelo Programa de Pós-graduação em Engenharia Elétrica do Departamento de Engenharia Elétrica do Centro Técnico Científico da PUC-Rio. Aprovada pela Comissão Examinadora abaixo assinada.
\end{abstract}

\author{
Prof. Weiler Alves Finamore \\ Orientador \\ Departamento de Engenharia Elétrica - PUC-Rio
}

Prof. Raimundo Sampaio Neto

Departamento de Engenharia Elétrica - PUC-Rio

Prof. Marco Antonio Grivet Matoso Maia Departamento de Engenharia Elétrica - PUC-Rio

Prof. Valdemar Cardoso Da Rocha Júnior Departamento de Engenharia Elétrica - UFPE

Prof. José Eugenio Leal

Coordenador Setorial do Centro Técnico Científico - PUC-Rio

Rio de Janeiro, 03 de Julho de 2008 
Todos os direitos reservados. É proibida a reprodução total ou parcial do trabalho sem autorização da universidade, do autor e do orientador.

Franklin Antonio Sanchez Paiba

Graduou-se em Engenharia Electrónica na Pontifícia Universidade Católica del Perú (Lima, Perú).

Ficha Catalográfica Sanchez Paiba, Franklin Antonio

Códigos Fontanais Bidimensionais para Canais com Apagamento / Franklin Antonio Sanchez Paiba; orientador: Weiler Alves Finamore. - Rio de Janeiro : PUC-Rio, Departamento de Engenharia Elétrica, 2008.

v., 81 f: il. ; $29,7 \mathrm{~cm}$

1. Dissertação (mestrado) - Pontifícia Universidade Católica do Rio de Janeiro, Departamento de Engenharia Elétrica.

Inclui referências bibliográficas.

1. Engenharia Elétrica - Tese. 2. Códigos Fontanais. 3. Códigos LT. 4. Códigos Raptor. 5. Códigos com taxa versátil. 6. Canais com apagamento. 7. Distribuição de graus. I. Alves Finamore, Weiler. II. Pontifícia Universidade Católica do Rio de Janeiro. Departamento de Engenharia Elétrica. III. Título. 


\section{Agradecimentos}

A meu orientador Weiler Finamore pela amizade, paciência, compreensão, confiança e excelente orientação, que me motivaram para a realização do presente trabalho.

À CAPES e à PUC-Rio, pelos auxílios concedidos, sem os quais este trabalho não poderia ter sido realizado.

Aos professores do CETUC, em especial aos professores José Mauro e Raimundo, que agregaram conhecimento valioso em minha vida professional.

Aos meus pais, Giner e Yolanda, pela educação, atenção e carinho de todas as horas.

Aos meus irmaõs Karin e Ivan, pelo carinho, apoio, incentivo e por estar sempre presentes em minha vida.

A minha amiga Kelly, pela atenção, carinho e apoio incondicional em todo momento.

A meu amigo Humberto, pela ajuda, paciência e orientação.

Ao meus primos Gerardo, Sara e Jose Luis por todo apoio, paciência e compreensão.

Ao pessoal do CETUC, em particular ao Dick, Carlos, Marcelo e Tiago, pelo companheirismo e apoio em muitas ocasiões.

A todos os amigos e familiares que de uma forma ou de outra me estimularam ou me ajudaram. 


\section{Resumo}

Sanchez Paiba, Franklin Antonio; Alves Finamore, Weiler. Códigos Fontanais Bidimensionais para Canais com Apagamento. Rio de Janeiro, 2008. 81p. Dissertação de Mestrado - Departamento de Engenharia Elétrica, Pontifícia Universidade Católica do Rio de Janeiro.

Esta dissertação aborda o estudo de códigos fontanais (códigos LT e códigos Raptor) que são uma classe de códigos criados para a transmissão de dados de maneira confiável e eficiente através de canais os quais podem ser modelados como canais com apagamento. Os códigos LT e códigos Raptor são denominados códigos fontanais, devido a que eles são uma boa aproximação para o conceito de fontanas digitais. Além disso, eles são classificados como códigos de taxa versátil, no sentido que o número de símbolos codificados que podem ser gerados a partir dos dados de entrada é potencialmente ilimitado.

Códigos LT são capazes de recuperar, com probabilidade maior do que $(1-\delta)$, um conjunto de $k$ símbolos de entrada a partir de quaisquer $k+O\left(\sqrt{k} \ln ^{2}(k / \delta)\right)$ símbolos codificados recebidos, com uma média de $O(k \ln (k / \delta))$ operações $X O R$. Os códigos Raptor são uma extensão de códigos LT, na qual o processo de codificação é composto de duas etapas: um código de bloco de comprimento fixo (denominado pré-código) e um código LT com uma distribuição de graus apropriada.

Investigou-se o desempenho dos códigos LT usando duas novas distribuições de graus (Sóliton Robusta Melhorada e Sóliton Robusta Truncada) e foi proposto um modelo de códigos LT Bidimensionais, na qual os símbolos de entrada são agrupados em forma de matriz. Neste esquema os blocos correspondentes às linhas da matriz são codificados usando um código LT e, em seguida, a matriz resultante tem suas colunas também codificadas usando um código LT. Ainda que a complexidade do esquema tenha sido dobrada o desempenho alcançado pelos códigos LT Bidimensionais superou o desempenho dos códigos LT convencionais para situações em que a qualidade do canal $B E C$ é elevada.

\section{Palavras-chave}

Códigos Fontanais. Códigos LT. Códigos Raptor. Códigos com taxa versátil. Canais com apagamento. Distribuição de graus. 


\section{Abstract}

Sanchez Paiba, Franklin Antonio; Alves Finamore, Weiler. Bidimensional Fountain Codes for Erasure Channels. Rio de Janeiro, 2008. 81p. MsC Dissertation - Departamento de Engenharia Elétrica, Pontifícia Universidade Católica do Rio de Janeiro.

Fountain Codes (LT Codes and Raptor Codes) are a class of codes proposed to efficient and reliably transmit data through Erasure Channels. LT Codes and Raptor Codes are a good approximation to the concept of digital fountain and as such are named as fountain codes. They are said to be rateless codes in the sense that the number of symbols produced by the encoder could grow, potentially, to infinite.

With probability of success larger than $(1-\delta)$, a decoder of an LT code based scheme can recover the $k$ transmitted symbols from any received block of $k+O\left(\sqrt{k} \ln ^{2}(k / \delta)\right)$ correct symbols with an average of $O(k \ln (k / \delta)) X O R$ operations. Raptor codes are an extension of the LT codes idea, with a tandem scheme where a fixed length block code (namely a pre-code) is followed by an LT code that uses a properly chosen degree distribution.

In this dissertation the performance of LT codes with two recently proposed degree distributions, the Improved Robust Soliton and the Truncated Soliton Robust Distribution were investigated. A new scheme called Bidimensional LT Codes, has been proposed. In this scheme the input symbols are structured in a matrix form and afterwards the blocks corresponding to the lines of the matrix are encoded with an LT code. The columns of the new matrix so obtained are next encoded with a similar LT code. The complexity of the new scheme is doubled and yet its performance only just surpasses that of the conventional LT scheme for high quality $B E C$.

\section{Keywords}

Fountain Codes. LT Codes. Raptor Codes. Rateless Codes. Erasure Channels. Degree Distribution. 


\section{Sumário}

$\begin{array}{ll}\text { Sumário das notações } & 12\end{array}$

1 Introdução 13

1.1 Motivação 13

$\begin{array}{lll}1.2 & \text { Sistema de Comunicação } & 14\end{array}$

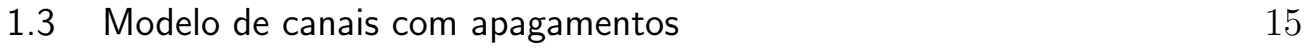

1.4 Fontanas Digitais 16

$\begin{array}{lll}1.5 & \text { Objetivos e organização do trabalho } & 18\end{array}$

2 Códigos LT 20

2.1 Codificação 21

2.2 Codificador LT como um código em grafo 23

$\begin{array}{ll}2.3 \text { Decodificação } & 25 \\ 2.3 .1 & 27\end{array}$

$\begin{array}{lll}2.3 .1 & \text { O processo LT } & 27\end{array}$

2.4 Distribuições de graus 30

2.4.1 Distribuição Sóliton Ideal 30

2.4.2 Distribuição Sóliton Robusta 30

2.5 Método das Transformações Inversas 33

2.5.1 Determinação do grau do símbolo codificado 33

2.6 Escolha dos parâmetros da distribuição Sóliton Robusta 34

2.6.1 Resultados obtidos das simulações 34

2.7 Análise de desempenho 37

2.7.1 Desempenho em canais ideais $\quad 37$

2.7.2 Desempenho em canais com apagamento 39

2.8 Comparação com códigos tradicionais para canais com apagamento 40

2.8.1 Códigos Reed-Solomon 41

2.8.2 Códigos Tornado 41

3 Projetando a distribuição de graus para códigos LT 43

3.1 Distribuição Sóliton Ideal 44

3.2 Distribuição Sóliton Robusta 46

3.2.1 Análise da distribuição Sóliton Robusta 49

4 Códigos LT Bidimensionais $\quad 52$

4.1 Distribuição de graus Sóliton Robusta Melhorada 52

4.2 Distribuição Sóliton Robusta Truncada para Códigos LT Sistemáticos 55

4.3 Esquema de códigos LT bidimensionais $\quad 57$

4.3.1 Codificação bidimensional 58

4.3.2 Decodificação bidimensional 58

$\begin{array}{ll}\text { 4.3.3 Configurações Utilizadas } & 59\end{array}$

$\begin{array}{lll}4.4 & \text { Resultados obtidos } & 59\end{array}$

5 Conclusões e Sugestões para trabalhos futuros $\quad 64$

5.1 Conclusões 64 
5.2 Sugestões para trabalhos futuros

Referências Bibliográficas

66

$\begin{array}{lll}\text { A Prova do Teorema } 3.1 & 69\end{array}$

B Códigos Raptor $\quad \mathbf{7 0}$

B.1 Códigos Raptor não-sistemáticos $\quad 70$

B.2 Codificação Raptor sistemática.

B.3 Construção de codificador e decodificador Raptor sistemático 74

B.4 Implementação dos códigos Raptor não-sistemáticos 75

B.4.1 Construção do pré-código LDPC 76

B.4.2 Construção do código LT interno 78

$\begin{array}{lll}\text { B.5 Resultados obtidos das simulações } & 79\end{array}$ 


\section{Lista de figuras}

1.1 Diagrama em Blocos de um Sistema de comunicações. 14

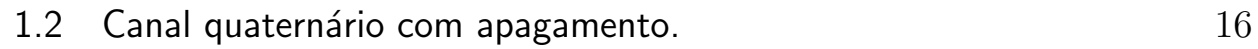

2.1 Grafo resultante da codificação. 24

2.2 Grafo do processo de decodificação 26

$\begin{array}{lll}2.3 & \text { Processo LT } & 29\end{array}$

2.4 A distribuição Sóliton Ideal $\rho(d)$ para o caso $k=10000$. 31

2.5 A distribuição Sóliton Ideal $\rho(d)$ e a função positiva $\tau(d)$ para o caso $k=10^{4}, c=0.2$ e $\delta=0.1$. 32

2.6 A distribuição Sóliton Robusta $\mu(d)$ para o caso $k=10^{4}, c=0.2$ e $\delta=0.1$. 32

2.7 Histograma do número de símbolos codificados necessários para a decodificação com sucesso para distintos valores de $c(k=1000$, $\delta=0.1)$.

2.8 Histograma do número de símbolos codificados necessários para a decodificação com sucesso para distintos valores de $\delta(k=1000$, $c=0.03)$.

2.9 Desempenho do código LT em canais ideais.

2.10 Sistema com códigos detetores de erros (CDE) usados em conjunto com códigos LT.

2.11 Desempenho do código LT em canais com apagamento.

3.1 Distribuição Sóliton Ideal: primeira iteração.

46

3.2 Distribuição Sóliton Ideal: segunda iteração.

3.3 Desempenho das distribuições Sóliton Robusta com parâmetros $c=0.03$ e $\delta=0.1$ e Sóliton Ideal.

3.4 Distribuição Sóliton Robusta: primeira iteração. 50

3.5 Distribuição Sóliton Robusta: segunda iteração. 50

4.1 A distribuição Sóliton Robusta Melhorada para o caso $k=10000$, $c=0.2$ e $\delta=0.1$

4.2 Histograma do número de símbolos necessários para a decodificação com sucesso de um código LT com distribuição Sóliton Robusta Melhorada $(k=1000, c=0.03$ e $\delta=0.1)$.

4.3 Histograma do número de símbolos necessários para a decodificação com sucesso de um código LT com distribuição Sóliton Robusta $(k=1000, c=0.03$ e $\delta=0.1)$.

4.4 Desempenho das distribuições Sóliton Robusta e Sóliton Robusta Melhorada para um código $\operatorname{LT}(1000,1300)$ com parâmetros $c=$ 0.03 e $\delta=0.1$.

4.5 A distribuição Sóliton Robusta Truncada $\Omega(d)$ para o caso $k=$ $10000, c=0.2, \delta=0.1$ e $\gamma=6$.

4.6 Reagrupamento bidimensional dos símbolos de entrada $s_{k}$.

4.7 Processo de codificação e decodificação bidimensional. 
4.8 Comparação de desempenho entre as configurações que usam a distribuição SR. Parâmetros utilizados: $k=1000, c=0.03, \delta=0.1$ e $\gamma=6$.

4.9 Overhead $(\epsilon)$ requerido para uma decodificação bem sucedida, em função de $P_{a}$. Parâmetros utilizados: $k=1000, c=0.03, \delta=0.1$ e $\gamma=6$.

4.10 Comparação de desempenho entre as configurações que usam a distribuição SRM. Parâmetros utilizados: $k=1000, c=0.03$, $\delta=0.1$ e $\gamma=6$.

4.11 Taxa de apagamento de símbolo em função da capacidade do canal $\left(1-P_{a}\right)$. Parâmetros utilizados: $k=1000, c=0.03, \delta=0.1, \gamma=6$ e $\epsilon=30 \%$.

B.1 Etapa de Pré-codificação em códigos Raptor. 71

B.2 Diagrama em bloco da codificação Raptor sistemática. 74

B.3 Restrições na codificação do código Raptor sistemático. 74

B.4 Diagrama em blocos do código Raptor. $\quad 75$

B.5 Processo de codificação do código Raptor.

B.6 Grafo de Tanner correspondente à matriz de paridade $\mathbf{H}$ de (B-13). 77

B.7 Probabilidade de falha em função do overhead. Canal ideal. $\quad 80$

B.8 Taxa de apagamento de símbolo em função do overhead. Canal $B E C$ com $P_{a}=0,04$

B.9 Comparação de desempenho entre códigos LT com distribuição SRM e códigos Raptor. Canal Ideal. 


\section{Lista de tabelas}

2.1 Processo de codificação. 24

2.2 Número médio de símbolos necessários à decodificação bem sucedida para distintos valores de $c(k=1000, \delta=0.1)$.

2.3 Número médio de símbolos necessários à decodificação bem sucedida para distintos valores de $\delta(k=1000, c=0.03)$.

2.4 Número médio de símbolos necessários à decodificação bem sucedida para distintos valores de $k(c=0.03, \delta=0.1)$ 


\section{Sumário das notações}

$\begin{array}{ll}k & \text { Número de símbolos de entrada } \\ n & \text { Número de símbolos codificados } \\ R & \text { Taxa do código } \\ \rho(d) & \text { Distribuição Sóliton Ideal } \\ \tau(d) & \text { Função positiva complementaria } \\ \mu(d) & \text { Distribuição Sóliton Robusta } \\ \bar{R} & \text { Número médio de símbolos codificados de grau um } \\ c & \text { Parâmetro da distribuição Sóliton Robusta } \\ \delta & \text { Limite superior da probabilidade de falha na decodificação de códigos LT } \\ \beta & \text { Constante de normalização } \\ P_{a} & \text { Probabilidade de apagamento do canal BEC } \\ (1+\epsilon) & \text { Overhead do código LT } \\ h_{t}(d) & \text { Número de símbolos codificados de grau } d \text { na t-ésima iteração } \\ \nu & \text { Fator complemento para obter a distribuição Sóliton Robusta Melhorada } \\ \mu(d, \gamma, \nu) & \text { Distribuição Sóliton Robusta Truncada } \\ \beta^{\prime} & \text { Constante de normalização } \\ \gamma & \text { Fator de truncamento } \\ \left(1+\varepsilon_{\text {Raptor }}\right) & \text { Overhead do código Raptor } \\ m & \text { Número de símbolos de paridade do pré-código LDPC } \\ w_{c} & \text { Peso das colunas da matriz de paridade H do código LDPC } \\ w_{r} & \text { Peso das linhas da matriz de paridade H do código LDPC } \\ L & \text { Número de símbolos pré-codificados do código Raptor } \\ \Omega_{D}(x) & \text { Distribuição de graus para códigos Raptor }\end{array}$

